\title{
RETRIEVAL OF THE TROPOSPHERIC AEROSOL MICROPHYSICAL CHARACTERISTICS FROM THE DATA OF MULTIFREQUENCY LIDAR SENSING
}

\author{
S. Samoilova, ${ }^{1}$ M. Sviridenkov, ${ }^{2}$ I. Penner, ${ }^{1, *}$ G. Kokhanenko, ${ }^{1}$ and Yu. Balin ${ }^{1}$ \\ IV.E. Zuev Institute of Atmospheric Optics, Tomsk 634055, Russia \\ 2A.M. Obukhov Institute of Atmospheric Physics, Moscow 119017, Russia \\ *Corresponding author: penner@iao.ru
}

\begin{abstract}
Regular lidar measurements of the vertical distribution of aerosol optical parameters are carried out in Tomsk $\left(56^{\circ} \mathrm{N}, 85^{\circ} \mathrm{E}\right)$ since April, 2011. We present the results of retrieval of microphysical characteristics from the data of measurements by means of Raman lidar in 2013. Section 2 is devoted to the theoretical aspects of retrieving the particle size distribution function $U(r)$ (SDF) assuming a known complex refractive index $m$ (CRI). It is shown that the coarse fraction cannot be retrieved unambiguously. When estimating $U(r)$ and $m$ together (section 3), the retrieved refractive index is non-linearly related to the optical coefficients and the distribution function, which leads to appearance of different, including false values of $m$. The corresponding $U(r)$ differs only slightly, so the inaccuracy in $m$ does not essentially affect the retrieval of the distribution function.
\end{abstract}

\section{INTRODUCTION}

At present, lidar systems are applied for routine observations in the European Aerosol Research LIdar NETwork (EARLINET) [1], the Asian Dust NETwork (AD-Net) [2], and the lidar network in CIS countries (CIS-LiNet) [3]. At night, they allow three backscattering coefficients $\beta\left(\lambda_{i}\right)$ to be evaluated for $\lambda_{i}=355,532$, and $1064 \mathrm{~nm}$ and two extinction coefficients $\sigma\left(\lambda_{k}\right)$ for $\lambda_{k}=355$ and $532 \mathrm{~nm}$, further referred to as $(3 \beta+2 \sigma)$. The algorithm used by us allows the errors to be decreased down to $\sim 5 \%(\sigma)$ and $\sim 2 \%(\beta)$, including the free troposphere. The spectral set of the optical coefficients and the errors of their estimation allow us to proceed to reconstruction of the vertical distribution of the microphysical aerosol characteristics.

\section{RETRIEVAL OF THE SIZE DISTRIBUTION FUNCTION UNDER THE ASSUMPTION OF KNOWN REFRACTIVE INDEX}

The determination of the particle size distribution function $U(r)$ (SDF) for the known complex refractive index $m$ (CRI) is reduced to the inversion of a system of linear algebraic equations. There is a problem for the lidar data: the parameters of the fine fraction are retrieved well for any regularization matrix, but the parameters of the coarse fraction are estimated ambiguously. The stabilizer order in fact specifies the range of the correct retrieval of the coarse aerosol mode parameters. This in turn leads to biased estimates of $U_{\text {coarse }}$. This clearly expressed dependence of the solution obtained on the restrictions on its smoothness is primarily caused by insufficient information, since for the coarse aerosol mode the spectral dependence of the optical coefficient becomes weak, and they contain in fact only two independent values.

From the mathematical point of view, the aerosol optical and microphysical characteristics are related with each other by the system of Fredholm's integral equations of the first kind:

$\int_{r_{\min }}^{r_{\max }} K_{j}(m, r) U(r) d \ln r+\varepsilon_{j}^{\text {meas }}=g_{j}^{\text {meas }}$,

where $g_{j}^{\text {meas }}$ are the extinction and backscattering coefficients measured at the corresponding wavelengths, $\varepsilon_{j}^{\text {meas }}$ are the measurement errors, $K_{j}(m, r)$ are the kernels of the equations depending on the refractive index and particle size. After the expansion, system of integral equations (1) is reduced to the system of linear algebraic equations for the vector of the weighting coefficients $\vec{u}=u_{k}$ : 


$$
A \vec{u}+\vec{\varepsilon}=\vec{g},
$$

where $\vec{\varepsilon}$ is the sum of experimental $\varepsilon_{j}^{\text {meas }}$ and mathematical $\varepsilon_{j}^{\text {math }}$ errors. Depending on the preliminary assumption about the expected properties of $u_{k}$, the regularized solution of Eq.

(2) can be found in the following form:

$\vec{u}=\left(A^{T} A+\alpha Q\right)^{-1} A^{T} \vec{g}$,

where $\alpha$ is the regularization parameter, and $Q$ is the smoothing matrix.

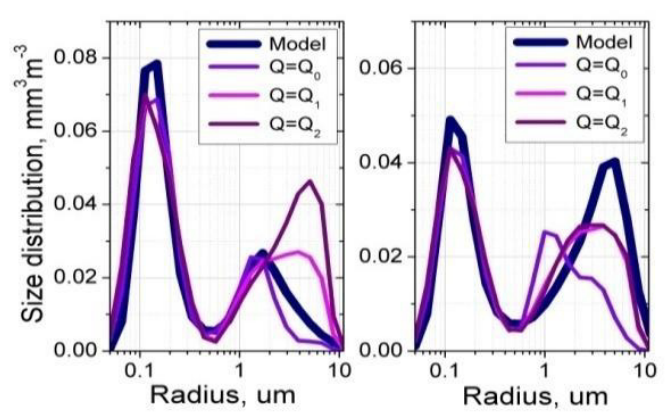

Figure 1. Examples of the SDFs reconstructed for two models; stabilizers of the zero $\left(\mathrm{Q}=\mathrm{Q}_{0}\right)$, first $\left(\mathrm{Q}=\mathrm{Q}_{1}\right)$ and second orders $\left(\mathrm{Q}=\mathrm{Q}_{2}\right)$ were used.

Figure 1 demonstrates the results of estimating the size distribution function by the Tikhonov method (3). The biased estimates of $U_{\text {coarse }}$ are predetermined by the a priori choice of the stabilizer order that in turn can lead to different values both on the abscissa and the ordinate, in particular, it fixes the geometrical mean radius of coarse particles $R_{\text {coarse }}$ (model $R_{\text {coarse }}^{\text {true }}$ and estimated $R_{\text {coarse }}^{\text {est }}$, see [4]). At the same time, the size distribution function of fine particles is retrieved well.

The statistical regularization method gives more accurate results:

$\vec{u}=\left(A^{T} A+W_{\varepsilon} W_{u}^{-1}\right)^{-1} A^{T} \vec{g}$,

where $\vec{u}$ is the sought vector of weight coefficients, and $W_{\varepsilon}, W_{u}$ are the covariance matrices of the errors, respectively. The matrix $W_{u}^{-1}$ is non-diagonal, and the preliminary estimation $W_{u}$ can make it possible to consider the data on the occurrence of the two aerosol modes (in fact, on the presence of several extrema), thereby cutting off a considerable number of physically unjustified solutions in the regularization stage.

Let us approximate the coarse $u_{k}$ by the polynomial $b_{0}+b_{1} \ln r+b_{2}(\ln r)^{2}$ for estimation $W_{u}$. Then Eq. (2) is rewritten in the form

$b_{0} \vec{l}_{0}+b_{1} \vec{l}_{1}+b_{2} \vec{l}_{2}=\vec{g}_{\text {coarse }}$,

where

$$
l_{0 k}=\sum A_{j k}, \quad l_{1 k}=\sum A_{j k} \ln r_{k},
$$

$l_{2 k}=\sum A_{j k}\left(\ln r_{k}\right)^{2}, \quad g_{\text {coarse }, j}=g_{j}-g_{\text {fine }, j} . \quad$ The solution of (5) for the coefficients $b_{i}$ is reduced to a solution of the system of three linear equations $B \vec{b}=\vec{d}$, where the components of the matrix $B$ and the vector $\vec{d}$ have the following forms: $B_{i k}=\sum l_{i j} l_{k j}, \quad d_{k}=\sum g_{\text {coarse }, j} l_{k j}$. For the known coefficients $b_{i}$, the mean radius is estimated from the formula $R_{\text {coarse }}^{\text {est }} \approx \exp \left(-0.5 b_{1} / b_{2}\right)$.

The accuracy of the estimation of $u_{k}$ is ambiguous: the estimate is correct up to $R_{\text {coarse }}^{\text {true }}=R_{\text {coarse }}^{\text {est }} \leq 3 \mathrm{um}$. It displaces toward higher values with increasing particle radius $R_{\text {coarse }}^{\text {est }}$, increases uncontrollably, and fails to be observed at $R_{\text {coarse }}^{\text {true }} \approx 4 u m$; then its displacement toward smaller values $R_{\text {coarse }}^{\text {est }}$ is observed. The instability of the estimate for $R_{\text {coarse }}^{\text {true }}>3 u m$ is also observed for other estimations of $u_{k}$; however, the boundaries of the range 3 and $4 \mathrm{um}$ are stable. Thus, $R_{\text {coarse }}^{\text {true }}=3 \mathrm{um}$ can be considered as an upper boundary of the correct estimation of the mean radius of the coarse fraction (and of the SDF as a whole and $\left.U(r)=\sum_{\text {finetcoarse }} B_{k}(r) u_{k}\right)$ from lidar measurements. However, the statistical regularization method allows application of the plausible $U(r)$ (plausible here implies the SDF 
retrieved previously from lidar or other measurements) for estimation of $W_{u}$. The results are presented in more details for 462 empirical; models obtained at the Zvenigorod AERONET site in 2011-2012 [4].

\section{SIMULTANEOUS RETRIEVAL OF THE SIZE DISTRIBUTION AND REFRACTIVE INDEX}

Although in practice $m$ and $U(r)$ are determined in parallel, there is a principle difference: CRI is a parameter of the kernel functions of the system of Fredholm's equations (1) and is not linearly related neither with the optical coefficients nor the distribution function. It makes the problem more complicated, because there are no standard methods for solving the non-linear inverse problems. It is not easy even to answer the question: is it always possible to estimate $m$ from lidar $(3 \beta+2 \sigma)$ measurements? The study of possible errors does not completely answer this question, because the errors can be caused both by the quality of the inversion algorithms and by efficiency of the used a priori data, and so on.

In general form, the problem is reduced to minimization of the discrepancy functional:

$$
\Phi(m)=\frac{1}{5} \sum_{j=1}^{5} \frac{\left|g_{j}^{\text {meas }}-g_{j}^{\text {calc }}(m)\right|}{g_{j}^{\text {meas }}} * 100 \% \text {. }
$$

On the one hand, it depends on the measured optical coefficients $g_{j}^{\text {meas }}$ (right part of Eq. (1)) and on the other hand on the same coefficients $g_{j}^{\text {calc }}(m)$, simulated for the all possible values of the real $m_{\text {real }}$ and imaginary $m_{\text {image }}$ parts of the refractive index. Application of iterative procedures like in [6] is unfounded, because a consequence of the undetermined data used is the solution dependence on the initial approximation. It is worthwhile to use the relative error in Eq. (6), because the coefficients $\sigma$ and $\beta$ can differ by two orders of magnitude.

$\Phi(m)$ values generate a complicated gully surface on the $\left(m_{\text {real }}, m_{\text {image }}\right)$ plane, and the low values areas are situated at the "bottom" of the gully at $\Phi(m) \leq \delta\left(\varepsilon^{\text {meas }}, \varepsilon^{\text {math }}\right) \approx 10 \%$. At $\varepsilon \neq 0$, the boundaries of the area lead to the appearance of the false local minima. Thus, in the presence of errors, multiple values of the refractive index on the $\left(m_{\text {real }}, m_{\text {image }}\right)$ plane correspond to the unique set of the values of the optical coefficients and distribution function too, and the problem of retrieving $m$ is undetermined even for a known SDF.
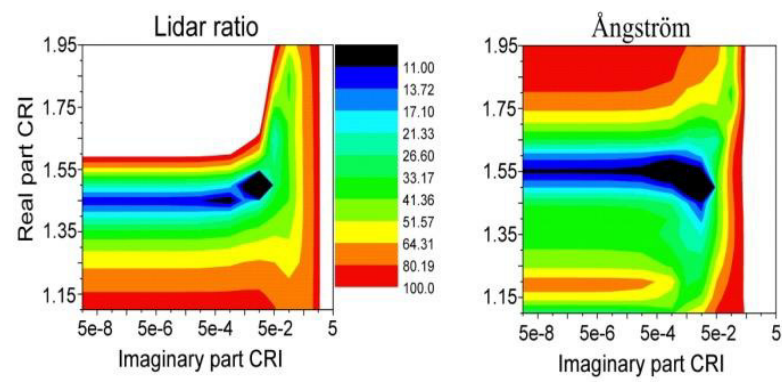

Figure 2. Different discrepancy functionals at the known SDF.

The calculation of $\Phi(m)$ for an already retrieved $U(r)$ is simpler. Two average discrepancy functionals are shown in Fig. 2: for the lidar ratio (left panel) and for the backscattering Angstrom exponent (right panel) obtained from measurements on September 24, 2013 (see details below). The first functional is analogous to Eq. (6) and leads to simultaneous increase of the real and imaginary parts of the refractive index for the minima of $\Phi(m)$. Increase of the imaginary part for decreasing real part is observed for the second functional, and the area of their crossing provides a unique value of CRI.

The results of the retrieval of the optical and microphysical characteristics from lidar data are shown in Fig. 3. Aerosol is transported in the boundary layer (from 0.5 up to $1.2 \mathrm{~km}$ ) and the middle troposphere (from 4.0 up to $5.3 \mathrm{~km}$ ) from Northern Europe, Atlantic Ocean, Canada with a north-westerly direction. Variations of the total to Rayleigh scattering ratio are shown in Fig. 3a. Parameters of large particles are not known exactly, since in the lower layer $R_{\text {coarse }}^{\text {est }}=9.08 \mathrm{um}$, and in the upper layer $R_{\text {coarse }}^{\text {est }}=4.79 u m$. So we assume that $R_{\text {coarse }}^{\text {true }} \in[3 \div 4 \mathrm{um}]$ and use the regularization matrix $Q=Q_{1}$ to obtain $W_{u}$ for 


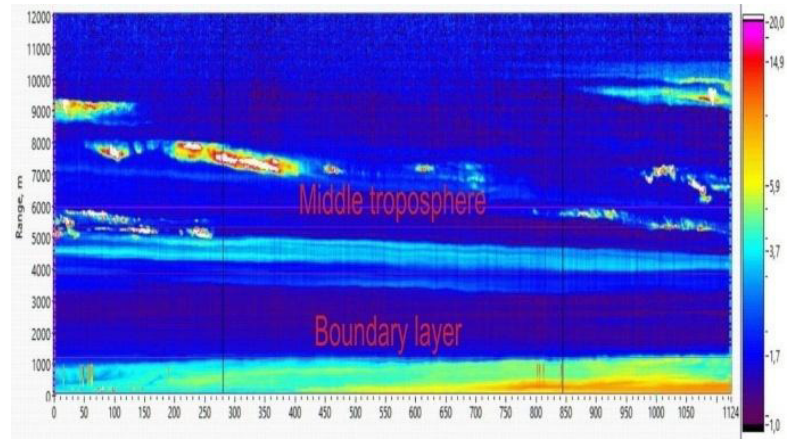

Figure 3a. Spatial-temporal variation of the scattering ratio at $1064 \mathrm{~nm}$, April 03, 2013.

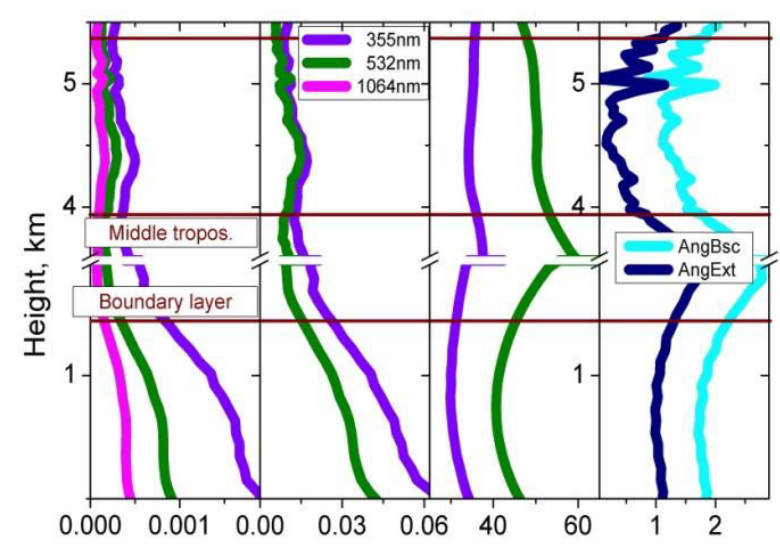

Figure 3b. Retrieval of the optical characteristics: backscatter (left panel), extinction (central left panel), lidar ratio (right central panel), Ångström exponent for $355 / 532$ (right panel).

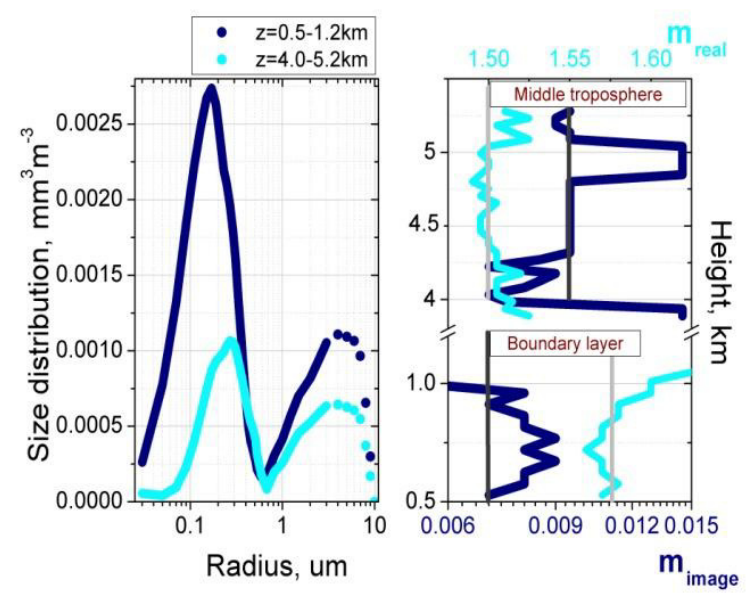

Figure 3c. Retrieval of SDF (left panel) and CRI (right panel) from lidar measurements on April 03, 2013. both layers. Average characteristics for the boundary layer are $m^{\text {mean }}=1.58+i 0.0065, R_{\text {fine }}=0.19 u m, R_{\text {coarse }}=4.0 u m$, and for the free troposphere $m^{\text {mean }}=1.50+i 0.009$, $R_{\text {fine }}=0.25 \mathrm{um}, R_{\text {coarse }}=3.5 \mathrm{um}$. However, possible ambiguity in the determination of the coarse particle parameters and possible false refractive index estimation have to be taken into account.

\section{ACKNOWLEDGEMENT}

This work was supported in part by the Russian Foundation for Basic Research, under grant N 1648-700307.

\section{REFERENCES}

[1] Pappalardo G., Amodeo A., Apituley A., et. al, 2014: EARLINET: towards an advanced sustainable European aerosol lidar network, Atmos. Meas. Tech., 7, 2389-2409, doi:10.5194/amt-7-2389-2014.

[2] Murayama T., Sugimoto N., Uno I., et al, 2001: Ground-based network observation of Asian dust events of April 1998 in east Asia, J. Geophys. Res., 106(D16), 18345-18359.

[3] Chaikovsky A.P., Ivanov A.P., Balin Yu.S., et al, 2006: CIS-LINET - Lidar Network for Monitoring Aerosol and Ozone in CIS Regions, Reviewed and Revised Papers Presented at the 23d ILRC, Nagasava C. and Sugimoto N., eds. (Nara, Japan), 671-672.

[4] Samoilova S.V., Sviridenkov M.A., and Penner I.E., 2016: Retrieval of the particle size distribution function from the data of lidar sensing under the assumption of known refractive index, Appl. Opt., 55(28), 8022-8029.

[5] Samoilova S.V., 2014: Retrieval of complex refractive index from lidar measurements: possibilities and limitations, Atmos. and Oceanic Optics, 27(3), 197-206.

[6] Dubovik O., M.D. King, 2000: A flexible inversion algorithm for retrieval of aerosol optical properties from Sun and sky radiance measurements, J. Geophys. Res., 105(D16), 20673 -20696 . 